\title{
Kostenübernahme nichtgelisteter Arzneimittel
}

Die Schweizerische Gesellschaft der Vertrauens- und Versicherungsärzte erarbeitete in Zusammenarbeit mit den Vertrauensärztlichen Diensten der grösseren Krankenversicherer eine Systematik zur allgemeinen Nutzenbewertung von Arzneimitteln. Anhand einheitlicher Kriterien sollen die Vertrauensärzte den Nutzen nichtgelisteter Arzneimittel rasch beurteilen können.

Max Giger,

Arthur Krähenbühl, Beat Seiler, Jürg Zollikofer
Korrespondenz:

Dr. med. Jürg Zollikofer Präsident Schweizerische Gesellschaft der Vertrauensund Versicherungsärzte SGV c/o MBC Markus Bonelli Consulting Wülflingerstrasse 59 CH-8400 Winterthu Tel. 0522260603 Fax 0522260604

info[at]vertrauensaerzte.ch
Die Spezialitätenliste (SL) [1] enthält beinahe alle in der ambulanten Therapie Erwachsener benötigten Arzneimittel. Diese Arzneimittel müssen gemäss der Fachinformation und allfälliger Limitationen (L) angewandt werden. Die Kosten der gelisteten Arzneimittel werden von der obligatorischen Krankenpflegeversicherung (OKP) übernommen.

In der Kinder- und Jugendmedizin müssen wegen fehlender klinischer Studien mit Patienten dieser Altersgruppe mehr als die Hälfte der Arzneimittel ausserhalb der Fachinformation (u.a. andere Dosierung, andere Applikation, andere Population)
Gesundheitsgesetzen nachvollziehbar festzuhalten. Vor dem Einsatz des Arzneimittels muss der Arzt beim Krankenversicherer die Kostengutsprache beantragen. Dies hat mittels eines medizinisch begründeten Gesuchs zuhanden des Vertrauensarztes zu erfolgen.

Im Prinzip werden von der OKP in begründeten Einzelfällen auch Arzneimittel ausserhalb der SL übernommen. Gemäss Artikel 71 a und b der Verordnung über die Krankenversicherung (KVV SR 832.102) übernimmt die OKP den Einsatz von Arzneimitteln unter folgenden Voraussetzungen:

\section{«Nichtgelistete Arzneimittel sollten einzig bei nachgewiesenem hohem} zu erwartendem medizinischem Nutzen empfohlen werden.»

angewandt werden. Auch im Bereich der Geriatrie und Alterspsychiatrie, insbesondere im Rahmen von Verhaltensstörungen bei Demenz, werden oft ältere Arzneimittel ausserhalb der Fachinformation angewandt. Der Einsatz ausserhalb der Fachinformation erfolgt aufgrund allgemeinen Wissens und Erfahrung sowie Guidelines [2], und die Kosten der Arzneimittel werden von den Krankenversicherern im Allgemeinen ohne Rückfrage übernommen.

Im Bereich der Onkologie und bei Behandlung seltener Krankheiten werden ältere und vor allem neuere Arzneimittel oft ausserhalb der Fachinformation (Off-Label-Use), ausserhalb der in der SL aufgeführten Limitatio (Off-Limitatio-Use), nichtgelistete registrierte Arzneimittel (Off-SL-Use) oder gar nicht registrierte Arzneimittel (Unlicensed-Use) angewandt. Seltener trifft dies für andere Therapiegebiete zu. Vorgängig der Medikation ausserhalb der Fachinformation oder gar nichtregistrierter Arzneimittel muss der Patient über die Ausnahmesituation orientiert werden [3]. Einzig bei expliziter Zustimmung des informierten Patienten dürfen diese Arzneimittel eingesetzt werden. In der Patientenakte ist der ausserordentliche Einsatz gemäss den kantonalen
- Das Arzneimittel ist eine unerlässliche Voraussetzung für die Durchführung einer anderen von der obligatorischen Krankenpflegeversicherung übernommenen Leistung

- Vom Einsatz des Arzneimittels wird ein grosser therapeutischer Nutzen gegen eine Krankheit erwartet, die für die versicherte Person tödlich verlaufen oder schwere und chronische gesundheitliche Beeinträchtigungen nach sich ziehen kann, und es ist keine andere wirksame und zugelassene Behandlungsmethode verfügbar.

Es ist Aufgabe des Vertrauensarztes, den Nutzen und die Zweckmässigkeit des Einsatzes beim individuellen Patienten zu beurteilen. Der Krankenversicherer bestimmt die Höhe der Vergütung.

Die Schweizerische Gesellschaft der Vertrauensund Versicherungsärzte (SGV) erarbeitete in Zusammenarbeit mit den Vertrauensärztlichen Diensten der grösseren Krankenversicherer eine Systematik zur allgemeinen Nutzenbewertung von Arzneimitteln [4]. Anhand einheitlicher Kriterien sollen die Vertrauensärzte den Nutzen der nichtgelisteten Arzneimittel innerhalb eines vertretbaren Zeitrahmens 
(20-30 Minuten) beurteilen können. Darauf basierend und unter Berücksichtigung wichtiger Prämissen des individuellen Patienten (u. a. klinischer Allgemeinzustand, Multimorbidität, aktuelle und frühere Behandlungen) können sie die Empfehlung zuhanden des Krankenversicherers formulieren. Zur Nutzenbeurteilung stehen heute die zwei auf den Abbildungen dargestellten Modelle zur Verfügung.

\section{9-Felder-Modell}

Der zu erwartende medizinische Nutzen des Arzneimittels wird aufgrund der wissenschaftlichen Evidenz und dessen Wirkung in den bekannten Studien ermittelt (Abb. 1). Die wissenschaftliche Evidenz wird in drei Stufen, von Case Reports bis Phase-III-Studien bzw. Registrierung durch EMA oder FDA, eingeteilt und auf der Ordinate dargestellt. Der methodologisch bedingten geringeren Evidenz im Falle eines Arzneimittels zur Behandlung einer seltenen Krankheit («Orphan Disease») wird mit einer um eine Stufe höheren Bewertung Rechnung getragen. Die wichtigsten Parameter, die in klinischen Studien zur Beurteilung der Wirkung der untersuchten Arzneimittel verwendet werden, sind als Mass des «medizinischen Effekts» auf der Abszisse aufgeführt. In der Onkologie sind dies das Gesamtüberleben ( Overall Survival»/OS) und die Zeitspanne zwischen dem Einsatz des Arzneimittels und dem Fortschreiten der Krankheit, d. h. das progressionsfreie Überleben («Progression-free Survival»/PFS). Das Ausmass des medizinischen Effektes wird in drei Stufen eingeteilt. Das Zusammenführen der wissenschaftlichen Evidenz mit dem medizinischen Effekt ermöglicht die Einteilung der Arzneimittel in vier Nutzenkategorien.

\section{MediScore-Modell}

Das MediScore-Modell (Abb. 2) ist eine Weiterentwicklung des Helsana-Modells zur Nutzenbestim- mung eines Arzneimittels [5]. Es ist das Ergebnis aus den Erfahrungen bei über 2000 Fällen. Es hat sich für die Anwendung onkologischer und nicht onkologischer Fälle bewährt. Das Modell gibt auch in OrphanSituationen eine zuverlässige Nutzenbewertung ab. Im Zentrum stehen die wissenschaftlich abgestützten Kriterien zur Datenqualität, zur Wirkung und zur Lebensqualität. Die Darstellung wurde vereinfacht und die Praxistauglichkeit verbessert. Die Kriterien basieren auf einem breiten Konsens, sie sind klar definiert und für jedermann nachvollziehbar. Neu wurde das Nebenwirkungsprofil (Adverse Events) detaillierter dargestellt und die Validität des Modells überprüft.

Beide Modelle werden von Vertrauensärzten angewandt. Sie erlauben eine Beurteilung des klinischen Nutzens innerhalb kurzer Zeit. Die Ergebnisse stimmen in hohem Ausmass überein. Es können beide Modelle «additiv» eingesetzt und die Resultate verglichen werden, um allfällige Unsicherheiten bei der Nutzenbeurteilung zu beseitigen.

Je besser die Wirkungen und Nebenwirkungen der Arzneimittel dokumentiert sind, desto weniger Unsicherheit tritt bei der Nutzenbeurteilung auf. Eine ausführliche und vertrauenswürdige Dokumentation - auch mit Resultaten aus Off-Label-Use erleichtert im Interesse der Patienten die Nutzenbeurteilung. Die Pharmaindustrie kann durch Kommunikation publizierter und nichtpublizierter Daten zu bekannten und neuen Wirkstoffen die Nutzenbeurteilung stärken und beschleunigen. Die möglichst breite Öffnung «schlummernder» bzw. «pharma-interner» Datenbanken für Vertrauensärzte und behandelnde Ärzte ist im Interesse einer sicheren und effizienten Patientenbehandlung. Dadurch werden weder der Off-Label-Use gefördert noch eine «Parallel-SL» geschaffen.

Die SGV begleitet im Rahmen einer Arbeitsgruppe den Einsatz dieser Modelle und veranlasst de-

Abbildung 1

9-Felder-Modell.

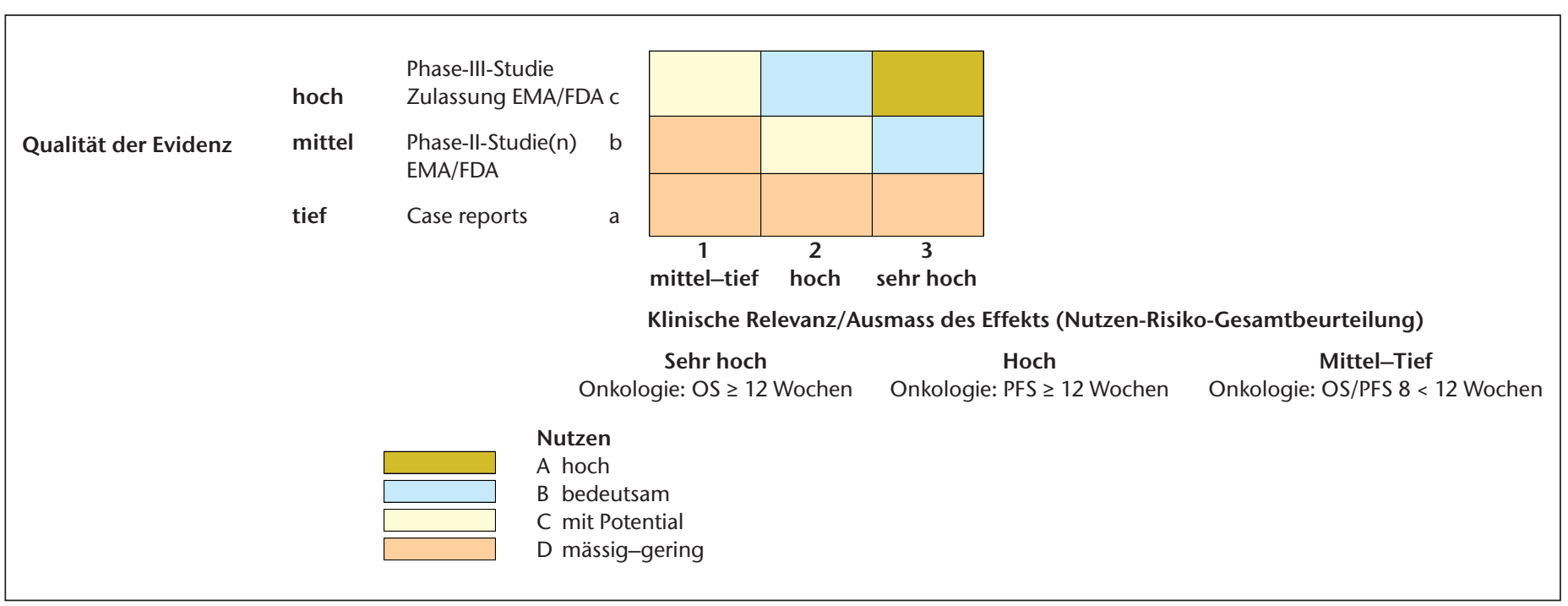




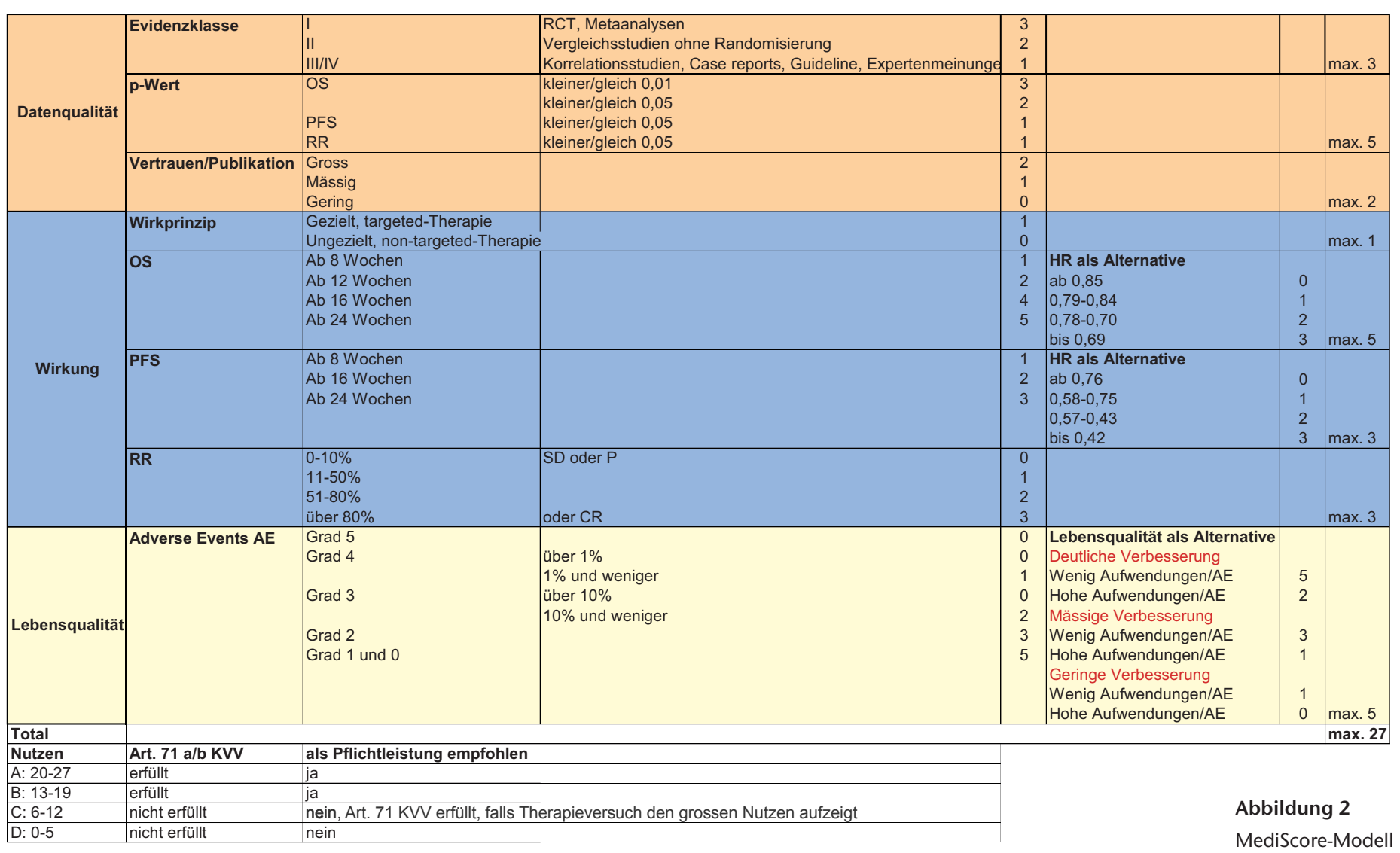

ren Anpassung an die Bedürfnisse der anwendenden Vertrauensärzte.

Im Prinzip gehören diese Modelle in die Hände der behandelnden Ärzte. Sie können dadurch in der Entscheidungsfindung und bei der Kommunikation mit den Patienten unterstützt werden. Ärzte sollten immer selbst vorgängig der Diskussion mit den betroffenen Patienten den Nutzen nichtgelisteter Arzneimittel abklären. Nichtgelistete Arzneimittel sollten einzig bei nachgewiesenem hohem zu erwartendem medizinischem Nutzen empfohlen werden. Dadurch wird die therapeutische Vereinbarung unterstützt. Die behandelnden Ärzte können das Ergebnis ihrer Nutzenbewertung dem Gesuch um Kostenübernahme beifügen. Dadurch kann der Prozess der Kostengutsprache effizienter gestaltet werden.

\section{Ausblick}

Am kommenden Jahreskongress der SGV in Fribourg (20./21.3.2013) werden weitere Workshops zur Nut- zenbewertung von Arzneimitteln insbesondere bei Off-Label-Use stattfinden. Die davon betroffenen Ärzte, Versicherer, Ämter und Pharmafirmen sind zur Teilnahme eingeladen.

\section{Literatur}

1 www.sl.bag.admin.ch

2 www.kinderdosierungen.ch

3 Giger M, Saxer U, Wildi A, Fritz MB. Medikationsprozess, in: Arzneimittelrecht. Zürich: Schulthess; 2013.

4 siehe www.vertrauensaerzte.ch $\rightarrow$ Fachliches $\rightarrow$ Empfehlungen $\rightarrow$ Nutzenbewertung

5 Seiler B, Fries R, Honegger H. Nutzenbewertung für Off-Label-Medikamente. Schweiz Ärztezeitung. 2012;93(19):723-5. 